Pacific Journal of Mathematics

SOME RANDOM FIXED POINT THEOREMS FOR
CONTINUOUS RANDOM OPERATORS 


\title{
SOME RANDOM FIXED POINT THEOREMS FOR CONTINUOUS RANDOM OPERATORS
}

\author{
SHIH-SEN CHANG
}

In this paper some random fixed point theorems for continuous random operators are obtained. These theorems generalize and unify some recent results.

1. Introduction. Random fixed point theorems are of fundamental importance in probabilistic functional analysis. In Polish spaces, i.e. separable complete metric spaces, random fixed point theorems were proved by Špaček, Hanš, Bharucha-Reid, Itoh, Engl and others (cf. $[1-6,8-11,14])$.

In this paper, we continue to consider this problem, and present several random fixed point theorems for abstract continuous random operators. These results generalize and unify some recent results.

2. Preliminaries. Throughout this paper, $(\Omega, \mathfrak{B}, P)$ denotes a complete probability measure space, $X$ is a separable complete metric space with metric $d$, and $(X, \mathfrak{X})$ is a measurable space, where $\mathfrak{X}$ is the $\sigma$-algebra of all Borel subsets of $X$.

A mapping $x: \Omega \rightarrow X$ is said to be a random variable with value in $X$, if the inverse image under the mapping $x$ of every Borel set of $X$ belongs to $\mathfrak{B}$. A mapping $T(\cdot, \cdot): \Omega \times X \rightarrow X$ is said to be a random operator, if $\{\omega \in \Omega: T(\omega, x) \in B\} \in \mathfrak{B}$ for all $x \in X, B \in \mathfrak{X}$. A random operator $T(\cdot, \cdot): \Omega \times X \rightarrow X$ is called continuous, if for each $\omega \in \Omega, T(\omega, \cdot)$ is continuous. A random variable $\xi: \Omega \rightarrow X$ is called a random fixed point of a random operator $T(\cdot, \cdot): \Omega \times X \rightarrow X$, if $T(\omega, \xi(\omega))=\xi(\omega)$ a.s.

LEMMA 1. ([12]). Let $x(\omega)$ be an $X$-valued random variable. Let $T: \Omega \times X \rightarrow X$ be a continuous random operator. Then $T(\omega, x(\omega))$ is also an $X$-valued random variable.

Let $T: \Omega \times X \rightarrow X$ be a continuous random operator. For any $X$-valued random variable $x(\omega)$ let $O_{T}(x(\omega), 0, \infty)$ denote the random orbit of $x(\omega) \in X$ under $T$, i.e.

$$
\begin{gathered}
O_{T}(x(\omega), 0, \infty)=\left\{x_{0}(\omega)=x(\omega), x_{1}(\omega)=T\left(\omega, x_{0}(\omega)\right) \ldots\right. \\
\left.x_{n}(\omega)=T\left(\omega, x_{n-1}(\omega)\right)=T^{n}\left(\omega, x_{0}(\omega)\right), \ldots\right\} .
\end{gathered}
$$

For any positive integers $i, j, j \geq i$, we put

$$
O_{T}(x(\omega), i, j)=\left\{x_{i}(\omega), x_{i+1}(\omega), \ldots, x_{j}(\omega)\right\} .
$$


For any pair of $X$-valued random variables $x(\omega), y(\omega)$, let $O_{T}(x(\omega), y(\omega), 0, \infty)$ denote the random orbit of $x(\omega), y(\omega)$ under $T$, i.e. $O_{T}(x(\omega), y(\omega), 0, \infty)$

$=\left\{x_{0}(\omega)=x(\omega), y_{0}(\omega)=y(\omega), x_{1}(\omega)=T\left(\omega, x_{0}(\omega)\right)\right.$,

$$
\begin{array}{r}
y_{1}(\omega)=T\left(\omega, y_{0}(\omega)\right), \ldots, x_{n}(\omega)=T\left(\omega, x_{n-1}(\omega)\right)=T^{n}\left(\omega, x_{0}(\omega)\right), \\
\left.y_{n}(\omega)=T\left(\omega, y_{n-1}(\omega)\right)=T^{n}\left(\omega, y_{0}(\omega)\right), \ldots\right\} .
\end{array}
$$

For any positive integer $i, j, j \geq i$, we denote

$$
\begin{aligned}
O_{T}(x(\omega), & y(\omega), i, j) \\
= & \left\{x_{l}(\omega), y_{i}(\omega), x_{i+1}(\omega), y_{i+1}(\omega), \ldots x_{j}(\omega), y_{j}(\omega)\right\} .
\end{aligned}
$$

For any subset $A \subset X$, let $\delta(A)$ be the diameter of $A$, i.e.

$$
\delta(A)=\sup _{x, y \in A} d(x, y) \text {. }
$$

\section{Main results.}

THEOREM 1. Let $T: \Omega \times X \rightarrow X$ be a continuous random operator. Suppose that there exists a positive integer $p$ such that for any $x \in X$ the following holds:

$$
\begin{aligned}
P\{\omega \in \Omega: & \delta\left(O_{T}(x, P, \infty)\right) \\
& \leq \Phi\left(\omega, \delta\left(O_{T}(x, 0, \infty)\right)\right\}=P\left(E_{x}\right)=1,
\end{aligned}
$$

where $\Phi(\omega, t): \Omega \times[0, \infty) \rightarrow[0, \infty)$ is a random function satisfying the following conditions

( $\Phi$ 1) For any fixed $\omega \in \Omega, \Phi(\omega, t)$ is non-decreasing and right continuous with respect to $t$.

( $\Phi$ 2) $P\{\omega \in \Omega: \lim (t-\Phi(\omega, t))=\infty\}=P(F)=1$.

(Ф 3) For any $t>\stackrel{t \rightarrow \infty}{0}$,

$$
P\left\{\omega \in \Omega: \lim _{n \rightarrow \infty} \Phi^{n}(\omega, t)=0\right\}=P\left(G_{t}\right)=1,
$$

where $\Phi^{n}(\omega, t)$ is the $n$th iterate of $\Phi(\omega, t)$.

Then for any random variable $x_{0}(\omega): \Omega \rightarrow X$ the sequence of the $X$-valued random variable $\left\{x_{n}(\omega)\right\}_{n=0}^{\infty}$ defined by

$$
x_{n}(\omega)=T\left(\omega, x_{n-1}(\omega)\right), \quad n=1,2, \ldots,
$$

converges almost surely to a random fixed point of $T$.

To prove Theorem 1, we first verify the following lemma. 
LEMMA 2. Let the random functions $\Phi(\omega, t)$ satisfy conditions $(\Phi 1)$ and (Ф 3). Then

(i) for any sequence $\left\{t_{n}(\omega)\right\}$ satisfying the following conditions:

(a) $t_{n}(\omega): \Omega \rightarrow[0, \infty), n=1,2, \ldots$,

(b) $t_{n}(\omega) \leq t_{n-1}(\omega), n=2,3, \ldots$,

(c) $P\left\{\omega \in \Omega: t_{n+1}(\omega) \leq \Phi\left(\omega, t_{n}(\omega)\right)\right\}=P\left(W_{n}\right)=1, n=1,2, \ldots$, we have

$$
\lim _{n \rightarrow \infty} t_{n}(\omega)=0 \quad \text { a.s.; }
$$

(ii) Particularly, if $t(\omega): \Omega \rightarrow[0, \infty)$ satisfies

$$
t(\omega) \leq \Phi(\omega, t(\omega)) \text { a. s. }
$$

then we have $t(\omega)=0 \mathrm{a}$. $\mathrm{s}$; and

(iii) for any $t>0$, we have $\Phi(\omega, t)<t$ a. s.

Proof. (i). By the right continuity of $\Phi(\omega, t)$ with respect to $t$ and condition (c), it is easty to see that the set

$$
W=\left(\bigcap_{n=1}^{\infty} W_{n}\right) \cap\left(\bigcap_{t>0}^{\infty} G_{t}\right) \in \mathfrak{B} \quad \text { and } \quad P(W)=1,
$$

where the sets $W_{n}, n=1,2, \ldots$, and $G_{t}$, respectively, are defined by condition (c) and ( $\Phi$ 3).

For an arbitrary, but fixed, $\tilde{\omega} \in W$ it follows from (c) that

$$
\begin{aligned}
0 & \leq t_{n+1}(\tilde{\omega}) \leq \Phi\left(\tilde{\omega}, t_{n}(\tilde{\omega})\right) \leq \Phi^{2}\left(\tilde{\omega}, t_{n-1}(\tilde{\omega})\right) \\
& \leq \cdots \leq \Phi^{n}\left(\tilde{\omega}, t_{1}(\tilde{\omega})\right) .
\end{aligned}
$$

Using conditions ( $\Phi$ 3) we have

$$
0 \leq \lim _{n \rightarrow \infty} t_{n+1}(\tilde{\omega}) \leq \lim _{n \rightarrow \infty} \Phi^{n}\left(\tilde{\omega}, t_{1}(\tilde{\omega})\right)=0 .
$$

Hence

$$
\lim _{n \rightarrow \infty} t_{n}(\omega)=0 \quad \text { a.s. }
$$

(ii) Putting $t_{n}(\omega)=t(\omega), n=1,2, \ldots$ in (i), we obtain immediately $t(\omega)=0$ a. .

(iii) Suppose that there exists some $t_{0}>0$ such that $t_{0} \leq \Phi\left(\omega, t_{0}\right)$ a. s. From (ii) we have $t_{0}=0$. This yields a contradiction. Hence the conclusion of (iii) holds.

This completes the proof of Lemma 2.

Now we return to the proof of Theorem 1. 
For any $X$-valued random variable $x_{0}(\omega)$, by the continuity of random operator $T$, it follows from Lemma 1 that the random orbit of $x_{0}(\omega)$ under $T$ i.e., $O_{T}\left(x_{0}(\omega), 0, \infty\right)=\left\{x_{n}(\omega)\right\}_{n=0}^{\infty}$,

$$
x_{n}(\omega)=T\left(\omega, x_{n-1}(\omega)\right), \quad n=1,2, \ldots
$$

is a sequence of $X$-valued random variables. By the separability of $X$, the continuity of $T(\omega, \cdot)$, and the right continuity of $\Phi(\omega, \cdot)$, it follows that the set

$$
H=\left\{\left(\bigcap_{x \in X} E_{x}\right) \cap F\left(\bigcap_{t>0} G_{t}\right)\right\} \in \mathfrak{B} \quad \text { and } P(H)=1 .
$$

By the assumptions of Theorem 1 , for the random variable $x_{0}(\omega) \in X$ we have

$$
\delta\left(O_{T}\left(x_{0}(\omega), p, \infty\right)\right) \leq \Phi\left(\omega, \delta\left(O_{T}\left(x_{0}(\omega), 0, \infty\right)\right)\right) \quad \forall \omega \in H
$$

It follows from (3.1) and (3.3) that

$$
\begin{aligned}
& \delta\left(O_{T}\left(x_{0}(\omega), n p, \infty\right)\right)=\delta\left(O_{T}\left(x_{(n-1) p}(\omega), p, \infty\right)\right) \\
& \quad \leq \Phi\left(\omega, \delta\left(O_{T}\left(x_{(n-1) p}(\omega), 0, \infty\right)\right)\right) \\
& \quad=\Phi\left(\omega, \delta\left(O_{T}\left(x_{0}(\omega),(n-1) p, \infty\right)\right)\right), \quad \omega \in H, n=1,2, \ldots
\end{aligned}
$$

However

$$
\begin{aligned}
& \delta\left(O_{T}\left(x_{0}(\omega),(n-1) p, \infty\right)\right) \\
& =\max \left\{\delta\left(O_{T}\left(x_{0}(\omega),(n-1) p, n p\right)\right),\right. \\
& \left.\delta\left(O_{T}\left(x_{0}(\omega), n p+1, \infty\right)\right), \sup _{\substack{(n-1) p \leq r \leq n p \\
s \geq n p+1}} d\left(x_{r}(\omega), x_{s}(\omega)\right)\right\} \\
& \leq \max \left\{\delta\left(O_{T}\left(x_{0}(\omega),(n-1) p, n p\right)\right), \delta\left(O_{T}\left(x_{0}(\omega), n p, \infty\right)\right),\right. \\
& \left.\sup _{(n-1) p \leq r \leq n p} d\left(x_{r}(\omega), x_{n p}(\omega)\right)+\sup _{s \geq n p+1} d\left(x_{n p}(\omega), x_{s}(\omega)\right)\right\} \\
& \leq \delta\left(O_{T}\left(x_{0}(\omega),(n-1) p, n p\right)\right)+\delta\left(O_{T}\left(x_{0}(\omega), n p, \infty\right)\right) .
\end{aligned}
$$

From (3.4) and (3.5) we obtain

$$
\begin{aligned}
& \delta\left(O_{T}\left(x_{0}(\omega),(n-1) p, \infty\right)\right) \\
& \leq \leq\left(O_{T}\left(x_{0}(\omega),(n-1) p, n p\right)\right) \\
& \quad+\Phi\left(\omega, \delta\left(O_{T}\left(x_{0}(\omega),(n-1) p, \infty\right)\right)\right), \quad \omega \in H .
\end{aligned}
$$


Taking $n=1$ in (3.6) we have

$$
\begin{aligned}
\delta\left(O_{T}(\right. & \left.\left.x_{0}(\omega), 0, \infty\right)\right) \\
\leq & \delta\left(O_{T}\left(x_{0}(\omega), 0, p\right)\right) \\
& +\Phi\left(\omega, \delta\left(O_{T}\left(x_{0}(\omega), 0, \infty\right)\right)\right), \quad \omega \in H .
\end{aligned}
$$

Now we prove that for $\omega \in H$

$$
\delta\left(O_{T}\left(x_{0}(\omega), 0, \infty\right)\right)<\infty .
$$

Suppose this is not the case, hence there exists some $\tilde{\omega} \in H$ such that

$$
\left.\delta\left(O_{T}\left(x_{0}(\omega), 0, \infty\right)\right)\right|_{\omega=\tilde{\omega}}=\infty .
$$

Letting

$$
A_{n}(\tilde{\omega})=\left.\delta\left(O_{T}\left(x_{0}(\omega), 0, n\right)\right)\right|_{\omega=\tilde{\omega}}, \quad n=1,2, \ldots
$$

we know that $\left\{A_{n}(\tilde{\omega})\right\}$ is an increasing sequence of positive numbers and

$$
\lim _{n \rightarrow \infty} A_{n}(\tilde{\omega})=\left.\delta\left(O_{T}\left(x_{0}(\omega), 0, \infty\right)\right)\right|_{\omega=\tilde{\omega}}=\infty .
$$

By condition ( $\Phi$ 2) and (3.7) we have

$$
\begin{aligned}
\infty & =\lim _{n \rightarrow \infty}\left(A_{n}(\tilde{\omega})-\Phi\left(\tilde{\omega}, A_{n}(\tilde{\omega})\right)\right. \\
& \leq\left.\delta\left(O_{T}\left(x_{0}(\omega), 0, p\right)\right)\right|_{\omega=\tilde{\omega}}<\infty .
\end{aligned}
$$

This yields a contradiction. Hence we obtain

$$
\delta\left(O_{T}\left(x_{0}(\omega), 0, \infty\right)\right)<\infty \quad \forall \omega \in H .
$$

\section{Putting}

$$
t_{n}(\omega)= \begin{cases}\delta\left(O_{T}\left(x_{0}(\omega), n p, \infty\right)\right) & \text { for } \omega \in H, \\ 0 & \text { for } \omega \in \Omega \backslash H, \quad n=0,1,2, \ldots\end{cases}
$$

from (3.4) we know that $\left\{t_{n}(\omega)\right\}$ satisfies all conditions of Lemma 2 (i). Therefore we have

$$
\lim _{n \rightarrow \infty} \delta\left(O_{T}\left(x_{0}(\omega), n p, \infty\right)\right)=0 \text { a.s. }
$$

This implies that $\left\{x_{n}(\omega)\right\}$ is a Cauchy sequence of $X$-valued random variables. Suppose $x_{n}(\omega) \rightarrow x_{*}(\omega)$ a. s. Hence $x_{*}(\omega)$ is also a $X$-valued random variable. Letting $n \rightarrow \infty$ in the following equality

$$
x_{n}(\omega)=T\left(\omega, x_{n-1}(\omega)\right) \text {, }
$$


and invoking the continuity of $T$, we have

$$
x_{*}(\omega)=T\left(\omega, x_{*}(\omega)\right) \text { a. s. }
$$

This completes the proof of Theorem 1 .

THEOREM 2. Let $\Phi(\omega, t)$ be the same as in Theorem 1 , and let $T$ be a continuous random operator from $\Omega \times X \rightarrow X$. Suppose that there exists a positive integer $p$ such that for any $k \in N$ ( $N$ denotes the set of all non-negative integers) and any $x \in X$ the following holds:

$$
\begin{aligned}
P\left\{\omega \in \Omega: d\left(T^{p}(\omega, x), T^{p+k}(\omega, x)\right)\right. \\
\left.\leq \Phi\left(\omega, \delta\left(O_{T}(x, 0, \infty)\right)\right)\right\}=P\left(E_{x k}\right)=1 .
\end{aligned}
$$

Then the conclusion of Theorem 1 still holds.

Proof. By the assumptions on $X, T$ and $\Phi$, it is easy to see that the set

$$
E=\bigcap_{x \in X} \bigcap_{k=0}^{\infty} E_{x k} \in \mathfrak{B} \text { and } P(E)=1 .
$$

Hence for any pair $r, s \in N$ (with no loss of generality we can assume $r \leq s$ ), and any $x \in X$, it follows from (3.8) that for any $\omega \in E$ the following holds:

$$
\begin{aligned}
d\left(T^{p+r}(\omega, x),\right. & \left.T^{p+s}(\omega, x)\right) \\
& =d\left(T^{p}\left(\omega, T^{r}(\omega, x)\right), T^{p+s-r}\left(\omega, T^{r}(\omega, x)\right)\right) \\
& \leq \Phi\left(\omega, \delta\left(O_{T}\left(T^{r}(\omega, x), 0, \infty\right)\right)\right) \\
& =\Phi\left(\omega, \delta\left(O_{T}(x, r, \infty)\right)\right) \\
& \leq \Phi\left(\omega, \delta\left(O_{T}(x, 0, \infty)\right)\right) .
\end{aligned}
$$

Consequently

$$
\begin{aligned}
\delta\left(O_{T}(x, p, \infty)\right) & =\sup _{r, s \geq 0} d\left(T^{p+r}(\omega, x), T^{p+s}(\omega, x)\right) \\
& \leq \Phi\left(\omega, \delta\left(O_{T}(x, 0, \infty)\right)\right), \quad \omega \in E
\end{aligned}
$$

i. e.

$$
\delta\left(O_{T}(x, p, \infty)\right) \leq \Phi\left(\omega, \delta\left(O_{T}(x, 0, \infty)\right)\right) \text { a. s. }
$$

This implies that from (3.8) we can deduce (3.1). Therefore the desired conclusion follows from Theorem 1. 
This completes the proof of Theorem 2 .

REMARK 1. In the deterministic case, the main result in Pal and Maiti [13] is a special case of Theorem 2.

As a corollary of Theorem 1 and Theorem 2 we have the following result.

COROllary 1. Let $T: \Omega \times X \rightarrow X$ be a continuous random operator. Suppose that there exists a positive integer $p$ such that for any $x \in X$ one of the following conditions holds:

$$
P\left\{\omega \in \Omega: \delta\left(O_{T}(x, p, \infty)\right) \leq \mathbb{Q}(\omega) \delta\left(O_{T}(x, 0, \infty)\right)\right\}=1 ;
$$

(3.10) for any $k \in N$

$$
P\left\{\omega \in \Omega: d\left(T^{p}(\omega, x), T^{p+k}(\omega, x)\right) \leq \mathbb{Q}(\omega) \delta\left(O_{T}(x, 0, \infty)\right)\right\}=1,
$$

where $\mathbb{Q}(\omega): \Omega \rightarrow[0, \infty)$ is a random variable satisfying

$$
P(\omega \in \Omega: 0<\mathbb{Q}(\omega)<1)=1 .
$$

Then the conclusion of Theorem 1 holds.

Proof. Taking $\Phi(\omega, t)=\mathscr{Q}(\omega) \cdot t, t \geq 0$, it is easy to see that in this case conditions ( $\Phi 1),\left(\begin{array}{ll}\Phi & 2\end{array}\right),\left(\begin{array}{l}\Phi \\ (3)\end{array}\right)$ are all satisfied, and the conclusion follows from Theorem 1 and Theorem 2 , respectively.

TheOREM 3. Let $T: \Omega \times X \rightarrow X$ be a continuous random operator. Let $\Phi$ be the same as in Theorem 1. Suppose that there exist positive integers $p, q$ such that for any pair $x, y \in X$

$$
\begin{aligned}
P\{\omega \in \Omega: & d\left(T^{p}(\omega, x), T^{q}(\omega, y)\right) \\
\leq & \left.\Phi\left(\omega, \delta\left(O_{T}(x, y, 0, \infty)\right)\right)\right\}=P\left(E_{x y}\right)=1 .
\end{aligned}
$$

Then $T$ has a unique $X$-valued random fixed point $x_{*}(\omega)$; and for any $X$-valued random variable $x_{0}(\omega)$ the sequence $\left\{x_{n}(\omega)\right\}$, where

$$
x_{n}(\omega)=T\left(\omega, x_{n-1}(\omega)\right), \quad n=1,2, \ldots,
$$

converges almost surely to $x_{*}(\omega)$.

Proof. With no loss of generality, we can assume $p \geq q$.

Considering the set $\tilde{G}=\bigcap_{x \in X} \bigcap_{y \in X}\left(E_{x y}\right)$, it is easy to see that $\tilde{G} \in \mathfrak{B}$ and $P(\tilde{G})=1$. 
For any $x \in X$, any $k \in N$ and any $\omega \in \tilde{G}$, we denote $y=$ $T^{p-q+k}(\omega, x)$. From (3.11) we have

$$
\begin{aligned}
d\left(T^{p}(\omega, x), T^{q}\right. & \left.\left(\omega, T^{p-q+k}(\omega, x)\right)\right) \\
& =d\left(T^{p}(\omega, x), T^{p+k}(\omega, x)\right) \\
& \leq \Phi\left(\omega, \delta\left(O_{T}\left(x, T^{p-q+k}(\omega, x), 0, \infty\right)\right)\right) \\
& =\Phi\left(\omega, \delta\left(O_{T}(x, 0, \infty)\right)\right) .
\end{aligned}
$$

By Theorem 1, for any random variable $x_{0}(\omega)$, the sequence of $X$-valued random variables $\left\{x_{n}(\omega)\right\}$ defined by

$$
x_{n}(\omega)=T\left(\omega, x_{n-1}(\omega)\right), \quad n=1,2, \ldots,
$$

converges almost surely to a random fixed point of $T$.

To prove $T$ has a unique $X$-valued random fixed point, we proceed as follows:

Let $x_{*}(\omega), \tilde{x}(\omega)$ be two $X$-valued random fixed points of $T$; therefore

$$
\begin{aligned}
d\left(x_{*}(\omega), \tilde{x}(\omega)\right) & =d\left(T^{p}\left(\omega, x_{*}(\omega)\right), T^{q}(\omega, \tilde{x}(\omega))\right) \\
& \leq \Phi\left(\omega, \delta\left(O_{T}\left(x_{*}(\omega), \tilde{x}(\omega), 0, \infty\right)\right)\right) \\
& =\Phi\left(\omega, d\left(x_{*}(\omega), \tilde{x}(\omega)\right)\right) \text { a.s. }
\end{aligned}
$$

Using Lemma 2(ii) we get $d\left(x_{*}(\omega), \tilde{x}(\omega)\right)=0$ a.s., i.e. $x_{*}(\omega)=\tilde{x}(\omega)$ a.s.

This completes the proof of Theorem 3 .

From Theorem 3, we can easily deduce the following corollaries.

Corollary 2. Let $T: \Omega \times X \rightarrow X$ be a continuous random operator. Suppose that there exist positive integers $p$ and $q$ such that for any pair $x, y \in X$

$$
P\left\{\omega \in \Omega, d\left(T^{p}(\omega, x), T^{q}(\omega, y)\right) \leq \mathbb{Q}(\omega) \delta\left(O_{T}(x, y, 0, \infty)\right)\right\}=1,
$$

where $\mathcal{Q}(\omega): \Omega \rightarrow[0, \infty)$ is a random variable such that

$$
P(\omega \in \Omega: 0<\mathscr{Q}(\omega)<1)=1 .
$$

Then the conclusion of Theorem 3 holds.

REMARK 2. In the deterministic case, Corollary 2 is a generalization of Fisher [7, Theorem 2]. 
Corollary 3. Let $T: \Omega \times X \rightarrow X$ be a continuous random operator. Suppose that there exist positive integers $p, q$ such that for any pair $x, y \in X$ (3.13) $P\left\{\omega \in \Omega: d\left(T^{p}(\omega, x), T^{q}(w, y)\right)\right.$

$$
\begin{aligned}
\leq \Phi\left(\omega, \max \left[d(x, y), d\left(x, T^{p}(\omega, x)\right)\right.\right. & \\
d\left(y, T^{q}(\omega, y)\right), & d\left(x, T^{q}(\omega, y)\right), \\
& \left.\left.\left.d\left(y, T^{p}(\omega, x)\right)\right]\right)\right\}=1 .
\end{aligned}
$$

Then the conclusion of Theorem 3 holds.

REMARK 3. Corollary 3 is a generalization of Theorem 4 in Chang [3] and Theorem 1 in [4].

In particular, from Theorem 3 we can obtain a deterministic result as follows:

Corollary 4. Let $(X, d)$ be a complete metric space, $T: X \rightarrow X a$ continuous mapping and $\Phi$ a function satisfying the following conditions:

(i) $\Phi:[0, \infty) \rightarrow[0, \infty)$ is nondecreasing and right continuous;

(ii) $\lim (t-\Phi(t))=\infty$;

(iii) $\stackrel{t \rightarrow \infty}{\text { For each } t>0}$

$$
\lim _{n \rightarrow \infty} \Phi^{n}(t)=0
$$

Suppose that there exist positive integers $p, q$ such that for any pair $x, y \in X$

$$
d\left(T^{p} x, T^{q} y\right) \leq \Phi\left(\delta\left(O_{T}(x, y, 0, \infty)\right)\right) .
$$

Then $T$ has a unique fixed point in $X$ and for any $x_{0} \in X$ the sequence $\left\{x_{n}=T^{n} x_{0}\right\}$ converges to this fixed point of $T$.

THEOREM 4. Let $T$ be a continuous random operator satisfying the following conditions:

$$
\begin{aligned}
& P\left\{\bigcup_{p=1}^{\infty} \bigcup_{q=1}^{\infty} \bigcap_{x \in X} \bigcap_{y \in X}[\omega\right. \in \Omega: d\left(T^{p}(\omega, x), T^{q}(\omega, y)\right) \\
&\left.\left.\leq \Phi\left(\omega, \delta\left(O_{T}(x, y, 0, \infty)\right)\right)\right]\right\}=P(Q)=1,
\end{aligned}
$$

where $\Phi(\omega, t)$ is the same as in Theorem 1.

Then the conclusion of Theorem 3 holds. 
Proof. By the assumptions of Theorem 4, it is clear that the set

$$
H_{1}=Q \cap F \cap\left(\bigcap_{t>0} G_{t}\right) \in \mathfrak{B} \text { and } P\left(H_{1}\right)=1,
$$

where the sets $Q, F, G_{t}$, respectively, are defined in (3.14), ( $\Phi$ 2) and ( $\Phi$ 3).

For an arbitrary, but fixed $\tilde{\omega} \in H_{1}$, hence $\tilde{\omega} \in Q$, there exist positive integers $p_{0}, q_{0}$ such that for any $x, y \in X$

$$
d\left(T^{p_{0}}(\tilde{\omega}, x), T^{q_{0}}(\tilde{\omega}, y)\right) \leq \Phi\left(\tilde{\omega}, \delta\left(O_{T}(x, y, 0, \infty)\right)\right) .
$$

By Corollary 4 there exists a unique fixed point $x_{*}(\tilde{\omega})$ of $T(\tilde{\omega}, \cdot)$. Taking any $X$-valued random variable $x_{0}(\omega)$, we define a sequence of $X$-valued random variables $\left\{x_{n}(\omega)\right\}$ :

$$
x_{n}(\omega)=T\left(\omega, x_{n-1}(\omega)\right), \quad n=1,2, \ldots .
$$

As $\omega=\tilde{\omega}$ by Corollary 4 we know that $x_{n}(\tilde{\omega})$ converges to $x_{*}(\tilde{\omega})$. This means that $x_{n}(\omega)$ converges almost surely to $x_{*}(\omega)$, hence $x_{*}(\omega)$ is also a $X$-valued random variable and

$$
x_{*}(\omega)=T\left(\omega, x_{*}(\omega)\right) \text { a.s. }
$$

This completes the proof of Theorem 4 .

REMARK 4. Theorem 6 of [3] is a special case of Theorem 4.

Especially, taking $p=q$ and $\Phi(\omega, t)=(1-1 / m) t$ in Theorem 4, where $m$ is some positive integer, we obtain a generalization of an important random fixed point theorem of Bharucha-Reid (cf. [1, Theorem $3,4]$ or [2, Theorem 6]). The main results in Hanš [8, 9] and Spaček [14] are also special cases of Theorem 4 .

\section{REFERENCES}

1. A. T. Bharucha-Reid, Random integral equations, Academic Press, New York, London, 1972.

2. __ Fixed point theorems in probabilistic analysis, Bull. Amer. Math. Soc., 83, No. 5 (1976), 641-657.

3. Shih-sen Chang, Random fixed point theorems in probabilistic analysis, Nonlinear Analysis, 5, No. 2 (1981), 113-122.

4. - On some fixed point theorems in probabilistic analysis $(I)$, J. of Sichuan University, Natural Science Edition, No. 3 (1980), 9-16.

5. H. W. Engl, A general stochastic fixed point theorem for continuous random operators on stochastic domains, J. Math. Anal. Appl., 66, No. 1 (1978), 220-231.

6 . Some random fixed point theorems for strict contractions and nonexpansive mappings, Nonlinear Analysis, 2, No. 5 (1978), 619-626.

7. B. Fisher, Quasi-contraction on metric space, Proc. Amer. Math. Soc., 75 (1979), 321-325. 
8. O. Hanš, Random fixed point theorems, in Transactions of the First Prague Conference on Information Theory, Statistical Decision Functions, Random processes, pp. 105-125, Publishing House of the Czechoslovak Academy of Sciences, Prague (1957).

9. _ـ_ Random operator equations, in proceedings of the 4th Berkeley Symposium on Mathematical Statistics and Probability, V. 2, part I, pp. 185-205, Univ. of California Press, Berkeley (1961).

10. S. Itoh, A random fixed point theorem for a multivalued contraction mapping, Pacific J. Math., 68 (1977), 85-90.

11. Random fixed point theorems with an application to random differential equation in Banach spaces, J. Math. Analysis Appl., 67 (2), (1979), 261-273.

12. R. Kannan and H. Salehi, Random nonlinear equations and monotonic nonlinearities, J. Math. Anal. Appl., 57 (1977), 234-256.

13. T. K. Pal and M. Maiti, Extensions of Ciric's quasi-contractions, Pure Appl. Math. Soc., 6 (1977), 17-20.

14. A. Špašk, Zuffallige gleichungen, Czech. Math. J., 5 (1955), 462-466.

Received May 28, 1981.

DEPARTMENT OF MATHEMATICS

SICHUAN UNIVERSITY

Chengdu, Sichuan 610064, China 



\section{PACIFIC JOURNAL OF MATHEMATICS}

EDITORS

Donald BABBITT (Managing Editor)

University of California

Los Angeles, CA 90024

Hugo Rossi

University of Utah

Salt Lake City, UT 84112

C. C. Moore and Arthur Ogus

University of California

Berkeley, CA 94720
J. DugunduI

Department of Mathematics

University of Southern California

Los Angeles, CA 90089-1113

R. FinN and H. SAmelson

Stanford University

Stanford, CA 94305

\section{ASSOCIATE EDITORS}
R. ARENS
E. F. BECKENBACH
B. H. NEUMANN
F. WolF
K. YoshidA (1906-1982)

\section{SUPPORTING INSTITUTIONS}

UNIVERSITY OF ARIZONA

UNIVERSITY OF BRITISH COLUMBIA

CALIFORNIA INSTITUTE OF TECHNOLOGY

UNIVERSITY OF CALIFORNIA

MONTANA STATE UNIVERSITY

UNIVERSITY OF NEVADA, RENO

NEW MEXICO STATE UNIVERSITY

OREGON STATE UNIVERSITY
UNIVERSITY OF OREGON

UNIVERSITY OF SOUTHERN CALIFORNIA

STANFORD UNIVERSITY

UNIVERSITY OF HAWAII

UNIVERSITY OF TOKYO

UNIVERSITY OF UTAH

WASHINGTON STATE UNIVERSITY

UNIVERSITY OF WASHINGTON 


\section{Pacific Journal of Mathematics}

Vol. 105, No. 1 September, 1983

Kenneth F. Andersen, On the transformation of Fourier coefficients of

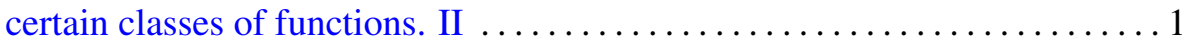

Gavin Brown, Irving Leonard Glicksberg and Edwin Hewitt, Indicator functions with large Fourier transforms $\ldots \ldots \ldots \ldots \ldots \ldots \ldots \ldots \ldots \ldots$

Shih-Sen Chang, Some random fixed point theorems for continuous random

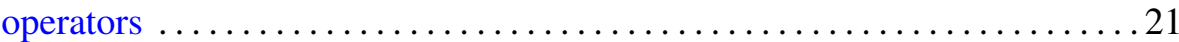

K. C. Chattopadhyay and Olav Njstad, Quasiregular nearness spaces and

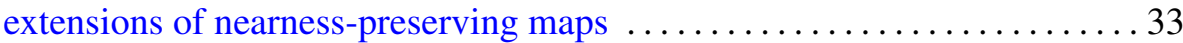

Thomas W. Cusick, The two-dimensional Diophantine approximation

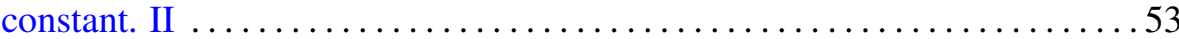

Eric Karel van Douwen and Jan van Mill, Spaces without remote points . . .669 Hector O. Fattorini, Convergence and approximation theorems for

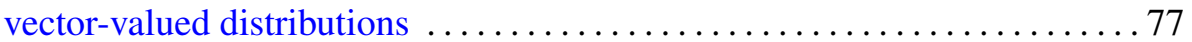

John J. F. Fournier and Louis Pigno, Analytic and arithmetic properties of

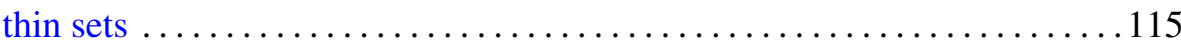

David Goss, On a new type of $L$-function for algebraic curves over finite

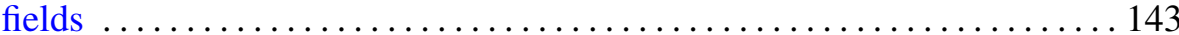

Douglas Austin Hensley, Lattice vertex polytopes with interior lattice

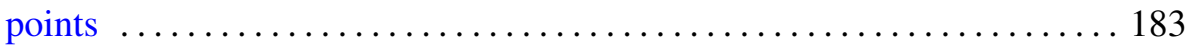

J. K. Kohli, Monotone extensions of mappings and their applications ...... 193

John C. Morgan, II, On equivalent category bases . . . . . . . . . . . . 207

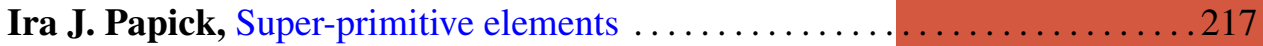

José Luis Rubio de Francia and José Luis Torrea, Vector extensions of

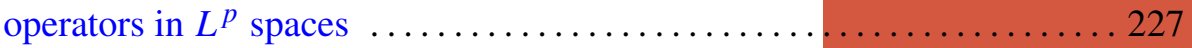

Mark Phillip Thomas, Closed ideals of $l^{1}\left(\omega_{n}\right)$ when $\left\{\omega_{n}\right\}$ is star-shaped $\ldots 237$ 